\title{
Re-exploring the Sentence-final Particle "LE" in the Propositional Domain, Epistemic Domain and Dialogic Domain
}

\author{
DENG Yu-yang \\ Niigata University, Niigata, Japan \\ Guangdong University of Foreign Studies South China Business College, Guangzhou, China
}

\begin{abstract}
Previous studies interpreting the meanings of the sentence-final particle "LE" displayed two trends: either excessively complicated or excessively general. Since some scholars established a theoretical foundation of propositional domain, epistemic domain and dialogic domain for the sentence-final particle "LE”, the nature or orientation of its semantic property has become more clear. However, there are also defects in the current "Three Domains" research model. In the first place, this model defines the meanings of the sentence-final particle "LE" as “emergence of new propositional content”, “emergence of new epistemic content”, and "emergence of new dialogic content”. But, the definition is excessively abstract and extensive. As many sentences not concluded with the particle "LE" can also express the three mentioned meanings, it fails to explain the difference between sentences ended with particle "LE" and ones without it. Secondly, the model fails to explore and discuss the nature or generation mechanism of relevant meanings of the particle "LE”. This study attempts to find a practical solution to those defects.
\end{abstract}

Keywords: sentence-final particle “LE”, propositional domain, epistemic domain, dialogic domain

\section{Introduction}

According to Xiao and Shen (2009), the propositional domain reflects the phenomenon of a certain kind of behavior, event or state emerged in the objective world; the epistemic domain indicates the phenomenon of a certain kind of perception or consciousness emerged in the subjective world; and the dialogic domain displays the phenomenon of a speaker sending direct or indirect requests to an addressee at the linguistic behavior level. That is, we can identify the nature of the meaning of a particle "LE" concluding a sentence based on the "Three Domains"research model, no matter what kinds of meaning it represents: they may reflect an objective development at propositional level; may refer to a subjective perception; or may represent a linguistic behavior. To put it simply, this study will call the sentence-final particle "LEs" in the propositional domain, epistemic domain and dialogic domain as "LE<propositional>”, "LE<epistemic $>$ ” and "LE<dialogic >” respectively.

DENG Yu-yang, Ph.D. in linguistics, Graduate School of Modern Society and Culture, Niigata University, Niigata, Japan; teacher of Guangdong University of Foreign Studies South China Business College, Guangzhou, China. 


\section{On the Meaning of “LE<propositional $>$ ” and Its Nature}

When mentioning the meaning of "LE<propositional>”, the following key words cannot be avoided: “shift”, “occurrence”, “emergence” and “realization” (Liu, 1998, pp. 35-48; Lü, 2010, pp. 548-556).

Among the plural meanings of "LE<propositional>” mentioned in foreign students-oriented Chinese textbooks used in Beijing Language and Culture University for each year, the "shift” and "emergence” have remained unchanged while other meanings have underwent constant modifications (Lü, 2010, pp. 548-556) Therefore, it can be said that the meaning "shift” and “emergence” have currently been widely accepted.

Shen (1987, pp. 4-19), Tan (2003, pp. 73-80) and relevant scholars chose the meaning "shift” for particle LE. For example, “(1) He has become a doctor (+LE).” This sentence clearly signals a shift: from a state of "he was not a doctor (but a writer)" to another state of "he is doctor."

Chen (2007, pp. 54-60), Sugimura (2009, pp. 1-12), Zhou and Ou (2014, pp. 8-15) and relevant scholars argued that examples, like “(2) It rained that night (+LE)” that reflects a natural phenomenon, do not prominently signal any “shift”. According to Sugimura (2009, pp. 1-12), Zhu (2016, p. 209) and relevant scholars, we can use "emergence” to describe the event in (2); by views from Jing (1993, pp. 52-57), Wang (1997, pp. 171-187), Oota (2003, p. 350) and relevant scholars, we can also employ “realization” to describe it; and from Zhang (2014, p. 24), Liu, Pan, and Gu (2016, p. 379) and relevant scholars, we can state it with the word "occurrence".

Liu (1998, pp. 35-48) pointed out that the so called "emergence” and "occurrence” are just "different in their use” and they are not “opposite concepts”; Xiao and Shen (2009, pp. 518-527), Lu (2014, pp. 43-47) thought that "emergence” and "occurrence” are essentially identical; Liu, Pan and Gu (2016, p. 379) argued that the meaning of "realization” is "becoming reality”, namely "occurrence” and “emergence”. In general, the so called “occurrence”, “emergence”, and "realization” essentially display some kind of commonality. To put it consistently, this study will use "occurrence" to summarize the meaning of "LE<propositional>” in the example (2).

Many scholars mentioned that "LE<propositional>” presents the function of shift on a tipping point ${ }^{1}$. This function itself implies some kind of "shift". But why "LE<propositional>” in the example (1) can signal clearly some kind of "shift" while the same one in the example (2) fails to bring the same effect? Sugimura (2009, pp. 1-12) figured out the cause of this result: "Is the shift mentioned here used as a term in the Chinese grammar that possesses a certain grammatical meaning or as a word with a common meaning?” This means that at the semantic level, "LE<propositional>” in the example (1) actually has the function of "shift" (shift on a tipping point), but does not display this "shift" at the semantic level. Because of this, this study will treat "shift" as a concept at the grammatical level. To avoid confusion between this concept and the "shift” at the semantic level, this study call the latter as "replacement".

So far, this study has divided the meanings of "LE<propositional>” into two categories: “occurrence” and "replacement".

Based on the meaning of the examples (1) and (2), this study summarizes “occurrence” and "replacement” as follows:

\footnotetext{
1 “Transformation of state” by Lu, F. B. (2002, pp. 109-118), “change of state” by Tan, C. J. (2004, pp. 26-31), “arrival of limit” by Liu, C. W. (2006, pp. 35-53), “bounded change” by Zhang, L. (2010, pp. 12-21), and “bounded” by Lü, W. G. (2007, pp. 147-149) all describe a phenomenon of change occurred at a limit.
} 
"Occurrence" means an event in the objective world comes into "being” from "not-being". This phenomenon only presents clearly a result of "being", but not the "not-being". This result of "being" of the event refers to "occurrence" of it, and displays the dynamic characteristic of instantaneity. The "not-being" of the event is its state before "occurrence". Formally, the predicate of a sentence expressing the meaning of "occurrence" is mainly a verb of movement.

"Replacement" means that an old state becomes a new and different state in the objective world. This phenomenon implies an old state that contains certain contentexisted before. "State" indicated by "replacement" is a kind of inwardness or a course of action with durative characteristic and does not present clearly the dynamic characteristic of instantaneity. Formally, this state can be described by adjectives, nouns, adverbs, static verbs, verbs considered to be "static phase" ${ }^{2}$ and other words, and can also be demonstrated by verbal phrases implying "ongoing”.

In short, the nature of "occurrence" and "replacement" lies in objectivity. Specifically, both meanings express a certain kind of development occurred at the objective and propositional level.

"Occurrence” and "replacement” defined in this study reflect the major views associated with the definitions of the sentence-final particle "LE". They can also embody the abstract concept- "emergence of new propositional content”.

\section{On Meaning of "LE<epistemic>” and Its Nature}

Large amount of research material argued that the sentence-final particle "LE” can express "affirmation”, “determination” or “confirmation” (Wang, 1984, pp. 161-162; Yue, 1997, pp. 14-17; Wang, 2000, pp. 17-21; Wu, 2002, pp. 23-27; He, 2003, pp. 10-18; Peng \& Zhou, 2005, pp. 136-141; Liu, 2006, pp. 201-244; Ye, 2014, pp. 85-91; Lü, 2016, pp. 351-358). A large part of the research material places such ways of expression like "affirmation”, "determination” or "confirmation" at the highest semantic level of the sentence-final particle "LE" (Yue, 1997, pp. 14-17; Wang, 2000, pp. 17-21; Wu, 2002, pp. 23-27; Peng \& Zhou, 2005, pp. 136-141; Lü, 2016, pp. 351-358). As “affirmation”, “determination”, “confirmation” and the like express a kind of subjective consciousness or subjective perception, they are brought into the category of "LE<epistemic>" in this study. However, the current ways of expression such as "affirmation”, "determination" and "confirmation” fail to clearly summarize the meaning of "LE<epistemic $>$ ". For instance, "affirmation" can be understood as a concept that is opposite to "negation" and "query", but its semantic value implied in the sentence-final particle "LE" does not intend to be opposite to "negation" and "query"; "determination” represents a kind of will, but its semantic value implied in the sentence-final particle "LE” does not intend to signal a will; and "confirmation" represents a kind of uncertainty, but its semantic value implied in the sentence-final particle "LE" does not represent such uncertainty. Purely in respect of language sense, the sentence-final particle "LE" indeed contains some kind of abstract "settling down". This meaning has been also considered as an "elusive conforming mood" (Wang, Li, \& Yue, 2009, pp. 312-333). As most studies gave an excessively general position to such "affirmation", "determination" or "confirmation", people often consider these moods are similar to "judgment" and "statement", resulting in the loss of the original value of "affirmation”, “determination” and "confirmation”.

\footnotetext{
2 According to Zhu, J. Z. (2000, pp. 26-28), the "static phase" of a verb means a kind of inwardness similar to meaning in dictionaries displayed by itself, or some kind of phenomenon and state it represents.
} 
This study summarizes the meaning of "LE<epistemic>" as "for certain", and considers "affirmation", "determination" or "confirmation" as the pragmatic effect of "LE<epistemic>" in the practical uses.

Among the previous studies, onlystudies by Liu (2006, pp. 201-244) gave detailed research on the nature of "affirmation”, “determination” or "confirmation” implied in "LE<epistemic>”, i.e. the nature of "for certain”. Liu (2006, pp. 201-244) argued that "for certain" implied in the sentence-final particle "LE” follows a course of "metal scanning”: this "for certain” is not made in one single step, but undergoes a process of psychological “exploration” or "reselection". In short, it is just because there are different options in one’s mind, so they need to "scan" these options and give "for certain" to one certain option. It can be said that the nature of "for certain" lies in subjectivity. Specifically speaking, it is a kind of consciousness of selection among subjective consciousnesses.

"For certain" defined in this study gives an explanation of the source of "affirmation”, “determination” or “confirmation”. It also embodies the abstract concept—-emergence of new epistemic content”.

\section{On Meaning of “LE<dialogic>” and Its Nature}

Tang (2015, p. 323) pointed out that components outside subject-predicate structure, especially particles at the both side of the structure, are in a position called "periphery". The sentence-final particle "LE" stays at the right side of subject-predicate structure and then displays the characteristic of "right periphery" proposed by Beeching and Detges (2014, p. 11). According to Beeching and Detges (2014, p. 11), the right periphery presents the feature of "intersubjective”. Sawada, Onodera, and Tousen (2017, pp. 3-51) analyzed the connotation of subjectivity and intersubjective and their relation via the dialogical relationship: subjectivity refers to the view, judgment, and comment of a speaker, putting emphasis on the speaker; intersubjective means a kind of subjectivity expressed by a speaker when regarding his addressee as the object of his consciousness (an object taken into account), putting emphasis on the dialogical relation between the two; and intersubjective is derived from subjectivity. In addition, it has been mentioned above that the dialogic domain displays the phenomenon of a speaker sending direct or indirect requests to an addressee at the linguistic behavior level. Therefore, "LE $<$ dialogic $>$ " presents the characteristic of subjective. Based on the definition of intersubjective made by Sawada, Onodera, and Tousen (2017, pp. 3-51), this study argued that the dialogic domain of the sentence-final particle "LE”'refers to not only the linguistic behavior proposed by Xiao and Shen (2009), but also the dialogical relation or consciousness of conversation between a speaker and his addressee.

To display both the linguistic behavior and the dialogical relation or consciousness of conversation, this study defines the meaning of the sentence-final particle "LE<dialogic>" as "request to listen (please listen to me)".

In "request to listen (please listen to me)", first of all, the imperative mood of "I request you" can express the subjectivity of a speaker ("I"), and can also reflect the relation between the speaker ("I") and his addressee (“you”); secondly, "please listen” presents the two kinds of linguistic behaviors: “listen” and "speak”, and thus complies with the "dialogue" in dialogic domain. In short, the nature of "request to listen (please listen to me)" lies in the intersubjective. Specifically, it refers to the consciousness of conversation among subjective consciousness. 
Taking as basis the massive data and statistics, Wang, Li, and Yue (2009, pp. 312-333) proved that the sentence-final particle "LE" has the function of "subjective close-distance interaction". "Subjective close-distance interaction" refers to a subjective and dialogical interaction relation. Its function is exactly in compliance with the function of "LE $<$ dialogic $>$ ". Therefore, "request to listen (please listen to me)" defined in this study can explain LE's function of "subjective close-distance interaction". It also embodies the abstract concept-“emergence of new dialogic content”.

\section{Conclusion}

This study re-explores the meaning and nature or generation mechanism of the sentence-final particle "LE" in the propositional domain, epistemic domain and dialogic domain. To a certain extent, it remedies defects in the “Three Domains" research model for the sentence-final particle "LE". The sentence-final particle "LE” also presents other pragmatic effects, such as "reporting”, “conveying new information”, "request”, "reminding”, “deduction”, "summing up”, “random listing”, “supplementing” and "ending conversation”. Those effects are in fact related to the meanings of "LE<propositional>”, "LE<epistemic >” and "LE< dialogic>”, and their nature or generation mechanism. In addition, "currently relevant state” (Li, Thompson, \& Thompson, 1982, pp. 19-44), i.e.one type of tenses of the sentence-final particle "LE" is actually the tense of "LE<dialogic>" in this study. As for those issues, we will give details in other studies.

\section{References}

Beeching, K., \& Detges, U. (2014). Discourse functions at the left and right periphery: Crosslinguistic investigations of language use and language change. Leiden: Brill.

Chen, X. H. (2007). The grammatical meaning of LE1 and LE2. Language Teaching and Linguistic Studies, (5), 54-60.

He, W. B. (2003). On the subjectivity of the mood particle LE2. Studies in Language and Linguistics, (1), 10-18.

Jing, C. (1993). The grammatical meaning of the particle LE. Linguistic Researches, (1), 52-57.

Li, C. N., Thompson, S. A., \& Thompson, R. M. (1982). The discourse motivation for the perfect aspect: The mandarin particle LE. In P. J. Hopper (Ed.), Tense-aspect: Between semantics \& pragmatic (pp. 19-24). Amsterdam: John Benjamins.

Liu, X. N. (1998). Modern Chinese language research. Beijing: Beijing Language and Culture University Press.

Liu, C. W. (2006). The aspect and modality of Chinese. Osaka: Osaka University Press.

Liu, Y. H., Pan, W. Y., \& Gu, W. (2016). Shiyong Xiandai Hanyu Yufa. Beijing: The Commercial Press.

Lu, F. B. (2002). Explain the Chinese auxiliary "LE” from a new angle. Nankai Linguistics, (1), 109-118.

Lu, F. Z. (2014). A corpus-based study on the auxiliary word "LE”. Journal of Ningbo University, (4), 43-47.

Lü, W. G. (2007). The bounded function of LE. Journal of Hubei University of Economics, (3), 147-149.

Lü, W. H. (2010). On the teaching of LE. Chinese Teaching in the World, (4), 548-556.

Lü, S. X. (2016). Xiandai Hanyu Babaici. Beijing: The Commercial Press.

Oota, T. (2003). Chinese historical grammar. Beijing: Peking University Press.

Peng, X. C., \& Zhou, S. (2005). The grammatical meaning of LE2. Academic Exchange, (1), 136-141.

Sawada, J., Onodera, N. K., \& Tousen, Y. K. (2017). The basic knowledge of periphery. In N. K. Onodera (Ed.), Periphery: Where pragmatic meaning is negotiated (pp. 3-51). Tokyo: Hituzi Syobo.

Shen, K. M. (1987). A study of LE2. Language Teaching and Linguistic Studies, (2), 4-19.

Sugimura, H. (2009). Script theory and the usage of LE2. Research on Chinese as a Second Language, (1), 1-12.

Tan, C. J. (2003). How LE2 indicates “change”. Language Teaching and Linguistic Studies, (3), 73-80.

Tan, C. J. (2004). The syntax, semantics and pragmatics of sentences ended on “LE”. Chinese Language Learning, (2), 26-31.

Tang, S. W. (2015). Lectures on cantonese grammar. Hong Kong: The Commercial Press.

Wang, L. (1984). Wang Li's collection of works (Vol. I). Jinan: Shandong Education Press.

Wang, W. X. (1997). A study of modern Chinese grammar theory. Beijing: Language \& Cultural Press.

Wang, Y. (2000). Use mechanism of “LE” and relevant teaching strategy. Language Teaching and Linguistic Studies, (3), 17-21. 
Wang, H. J., Li, R., \&Yue, Y. (2009). “LE2” and the function of “subjective close-distance interaction”. Yuyanxue Luncong, (40), 312-333.

Wu, L. F. (2002). “LE1” and “LE2”. YuwenYanjiu, (1), 23-27.

Xiao, Z. Y., \& Shen, J. X. (2009). Three domains of the sentence-final particle LE2 in mandarin Chinese. Zhongguo Yuwen, (6), 518-527.

Ye, Q. (2014). A semantic interpretation of the imperfective structure “The First Person Pronoun+V+LE2”. Journal of College of Chinese Language and Culture of Jinan University, (3), 85-91.

Yue, Z. Q. (1997). Temporal and aspectal functions of “LE2” in “V(LE1)-Ct (LE2)”. Chinese Language Learning, (3), 14-17.

Zhang, L. C. (2014). A temporal-aspectual composition model for the Chinese perfective LE structures. Doctoral dissertation of Fudan University.

Zhang, L. (2010). A cognitive and typological explanation of "LE” in Chinese. Chinese Language Learning, (6), 12-21.

Zhou, X. B., \& Ou, Y. D. (2014). An investigation on the acquisition of Chinese particle LE2 of Japanese students. Journal of College of Chinese Language and Culture of Jinan University, (4), 8-15.

Zhu, D. X. (2016). Lectures on grammar. Beijing: The Commercial Press.

Zhu, J. Z. (2000). Some aspects of Chinese usage. Tokyo: Hakuteisha. 